\title{
Potential therapeutic in preeclampsia: effect of resveratrol on endothelial cells incubated with plasma from pregnant before clinical onset of disease
}

\author{
Valeria C Sandrim ${ }^{1}$, Mayara Dias-Caldeira ${ }^{1}$, Heloisa Bettiol $^{2}$, Viviane Cardoso ${ }^{2}$, Marco A Barbieri $^{2}$, \\ Ricardo C Cavalli ${ }^{3}$
}

${ }^{I}$ Pharmacology, UNESP, Brazil, ${ }^{2}$ Department of Pediatrics, Faculty of Medicine of Ribeirao Preto, University of Sao Paulo, Ribeirao Preto, Sao Paulo, Brazil, ${ }^{3}$ Departmento de Ginecologia e Obstetricia, Faculdade de Medicina de Ribeirao Preto, Universidade de Sao Paulo (FMRP-USP), Ribeirao Preto, Brazil

Background: Preeclampsia is the main cause of mother and fetus mortality worldwide. Treatment is done by management of blood pressure levels and seizures prevention; however, currently the definitive treatment is delivery. Resveratrol is a compound presented in high quantity in red grapes that contribute to healthy endothelial function. We are exploring an in vitro model of preeclampsia incubating plasma with endothelial cell cultures. However, in this study we collected samples before clinical onset of preeclampsia. This is very important because we can postulate that a dietary intervention in pregnant may reduce risk to develop preeclampsia. The aims were: to verify if resveratrol induces more production of NO, improve cell viability and modulate expression of heme oxygenase-1 (HO-1, cellular protective enzyme) in endothelial cells incubated with plasma from case and control pregnant. Methods: We collected blood at 20-25 weeks of gestation from matched healthy pregnant during all gestation $(\mathrm{HP}, \mathrm{n}=5)$ and pregnant who subsequently develop preeclampsia (case, $n=5)$. Blood was centrifuged and plasma was incubated $(10 \% \mathrm{v} / \mathrm{v})$ for 24 hours in endothelial cells (HUVECs) with or not $30 \mu$ trans-resveratrol. Cellular viability was measured using MTT assay, HO-1 by ELISA and nitrite (NO metabolite) by Griess. P value $<0.05$ was considered statistically different.

Results: We found similar levels of cell viability between case and control, supplemented or not with resveratrol (all $\mathrm{P}>0.05$ ). Regarding supernatant nitrite levels also we values similar were found between case and control, and the addition of resveratrol not improved nitrite production. Interestingly concerning HO-1 levels, we found that cultures incubated with plasma from case presented higher levels of HO-1 compared to control (figure). When resveratrol was added, this difference diminished, however, still plasma from case induce HO-1 expression in higher degree compared to control cultures (figure). Conclusion: Our study demonstrates that resveratrol reduce HO-1 expression in case cultures demonstrating a reduction of cell-stress. Therefore, our results suggest that a diet rich in resveratrol, such as red grape juice, could be indicated to preeclampsia prevention.

Funded by Sao Paulo Research Foundation (FAPESP, grant \#2015/20461-8), The Brazilian National Council for Scientific and Technological Development (CNPq, grant \#2014-5/305587). 